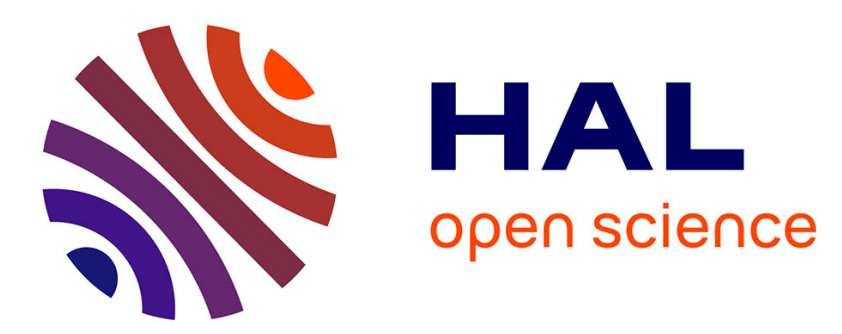

\title{
Investigation of modal interactions and their effects on the nonlinear dynamics of a periodic coupled pendulums chain
}

Diala Bitar, Najib Kacem, Noureddine Bouhaddi

\section{- To cite this version:}

Diala Bitar, Najib Kacem, Noureddine Bouhaddi. Investigation of modal interactions and their effects on the nonlinear dynamics of a periodic coupled pendulums chain. International Journal of Mechanical Sciences, 2017, 127, pp.130 - 141. hal-02131306

\section{HAL Id: hal-02131306 \\ https://hal.science/hal-02131306}

Submitted on 16 May 2019

HAL is a multi-disciplinary open access archive for the deposit and dissemination of scientific research documents, whether they are published or not. The documents may come from teaching and research institutions in France or abroad, or from public or private research centers.
L'archive ouverte pluridisciplinaire HAL, est destinée au dépôt et à la diffusion de documents scientifiques de niveau recherche, publiés ou non, émanant des établissements d'enseignement et de recherche français ou étrangers, des laboratoires publics ou privés. 


\title{
Investigation of modal interactions and their effects on the nonlinear dynamics of a periodic coupled pendulums chain
}

\author{
Diala Bitar, Najib Kacem, Noureddine Bouhaddi \\ FEMTO-ST Institute, UMR 6174, Department of Applied Mechanics, University of Bourgogne Franche-Comté, 24 rue de l'Épitaphe, F-25000 \\ Besançon, France.
}

\begin{abstract}
The nonlinear dynamics of a weakly coupled pendulums chain is investigated under primary resonance. The coupled equations governing the nonlinear vibrations are normalized and transformed into a set of coupled complex algebraic equations using the multiple scales method coupled with standing wave decomposition. A model reduction method is proposed to calculate the dominant dynamics without significant loss of accuracy compared to the full model. The validity of the proposed semi-analytical method is verified, and its role in identifying the type of the solution branches is highlighted. The modal interactions and their effects on the nonlinear dynamics are studied in the frequency domain in order to emphasize the large number of multimode solution branches and the bifurcation topology transfer between the modal intensities. Basins of attraction analysis have been performed, showing that the distribution of the multimodal solution branches generated by all modes collectively increases by increasing the number of coupled pendulums.
\end{abstract}

Keywords: Nonlinear dynamics, Periodic structures, Coupled pendulums, Modal interactions, Basins of attraction

\section{Introduction}

Nonlinear periodic systems are frequently encountered in serval scientific domains such as biology, optics, acoustics and mechanics. For instance, in the field of acoustics, Narisetti et al. [1] analyzed the influence of nonlinearity and wave amplitude on the dispersion properties of plane waves in nonlinear periodic materials, particularly in uniform granular media. In optics, Heinrich et al. [2] introduced optomechanical cells arrays as a new system to study the collective nonlinear dynamics. Moreover, in micro and nanotechnology, the collective dynamics of coupled nonlinear resonators array have been performed by Lifshitz et al. [3] using a discrete model.

In mechanics, the array of coupled pendulums represents a famous example of periodic nonlinear structures which can be described by the sine-Gordon model [4]. The latter is suitable for a large variety of physical systems, which explains the vast research area developed around this field. Several theorems on the existence of oscillatory, rotary,

\footnotetext{
Email addresses: diala.bitar@femto-st.fr(Diala Bitar), najib.kacem@femto-st.fr (Najib Kacem), noureddine.bouhaddi@femto-st.fr (Noureddine Bouhaddi)
} 
and mixed periodic motions of coupled pendulums were proved [5]. In addition, Cai et al. [6] identified two distinct regimes for a damped sine Gordon chain driven by the spatio-temporal periodic potential. Fradkov et et al. [7] developed a model for two-pendulum system and obtained from numerical solutions that both in- and anti-phase synchronizations are possible, depending on the initial conditions. Moreover, the synchronization phenomenon of two rotating parametric pendulums attached to common elastic support under harmonic excitation has been studied theoretically and experimentally [8]. The dynamics of the two- pendulum system has been investigated numerically and experimentally, due to the exhibited rich dynamical phenomena $[9,10]$. For a chain of coupled pendula driven periodically at one end, Khomeriki et al. [11] demonstrated experimentally the existence of a novel regime which produces an output frequency at an odd fraction of the driving frequency. In addition, it was shown that the bandwidth of absorber effectiveness can be increased substantially by using an array of pendulums with slightly different natural frequencies [12]. Recently, Liang et al. [13] reported an experiment using a coupled-pendulum chain to demonstrate various aspects of wave motion. Furthermore, Jallouli et al. [14] investigated the nonlinear dynamics of a 2D array of coupled pendulums under parametric excitation.

In other studies, Intrinsic Localized Modes (ILMs), existing as exact solutions in nonlinear lattices have been widely studied. Particularly, for an array of coupled pendulums [15] which is an ideal playground for showcasing many interesting features of discrete breathers, [16] which can be stabilized in the presence of driving and damping [17] or propagate energy in a forbidden band-gap [18]. The nonlinear coupling and decoupling between ILM and third allowed plane-wave mode, as well as both modes interaction with the driver, give rise to pulsating breather instability [19]. In addition, it was shown that the mass impurity in a parametrically driven, damped nonlinear coupled pendula has striking influence on the high-frequency modes [20]. Recently, The influence of adding external harmonic excitation on the intrinsic localized modes of coupled pendulums chains parametrically excited has been investigated [21]. Although the dynamics of coupled nonlinear pendulums was thoroughly investigated in the frequency and timespace domains, there is a real need to perform profound analysis of the collective dynamics of such systems in order to identify practical relations with the nonlinear energy localization phenomena in terms of modal interactions and bifurcation topologies [22].

In this paper, we propose a methodology based on an analytical-numerical solving procedure suitable for weekly coupled nonlinear pendulums. This methodology enables to perform profound analysis of complex model interactions in nonlinear periodic structures. The weakly coupled nonlinear equations have been derived and transformed into a set of coupled complex algebraic equations, using the multiple scales method and standing-wave decomposition, which are numerically solved. Numerical simulations have been performed in order to analyze the stability, the modal interactions and the bifurcation topologies resulting from the collective dynamics of three, six and twelve coupled pendulums. These proposed examples highlight the large number of multimodal solutions even for a small number of coupled pendulums. Based on standing wave mode projection, we propose a Reduced Order Model (ROM) approach that leads to an acceptable reduction ratio and a low computational cost compared to the full model. The complexity and the multivaludness of the responses were illustrated by a detailed study of the evolution of the basins of attraction 
with respect to the number of coupled pendulums.

\section{Complex amplitudes equations}

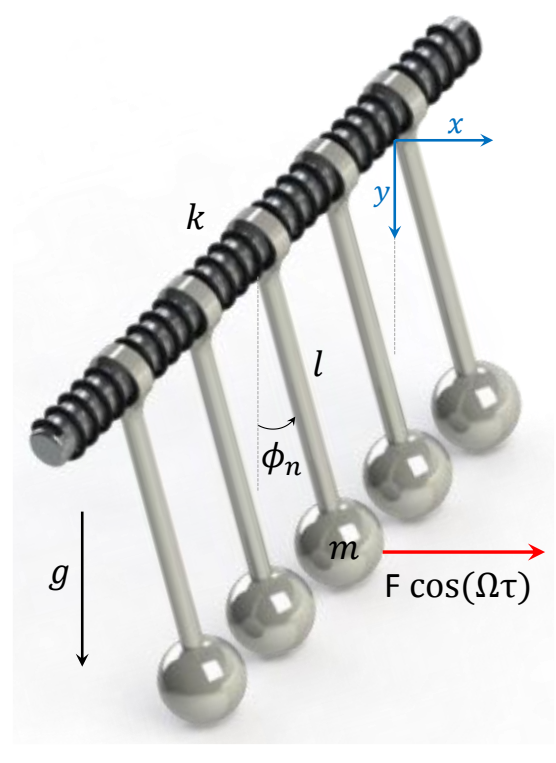

Figure 1: A chain of externally excited equidistant pendulums sharing a common axle. Each pendulum consist of a rigid rod, attached perpendicularly to the axle with a mass at the end. $k$ represents the linear coupling torque constant. Each pendulum is subjected to an external harmonic excitation $F \cos (\Omega \tau)$ and $\phi_{n}$ is the angle between the $n^{\text {th }}$ pendulum and the downward vertical.

Figure 1 shows the theoretical model of an array composed by an horizontal axle, containing $N$ identical pendulums separated by an equal distance. Each pendulum $n$ is attached perpendicularly to the axle, with a mass $m$ and length $l, \phi_{n}$ is the rotational degree-of-freedom (dof) of the pendulum measured from its stable equilibrium position. The pendulums " $n$ " and " $n+1$ " are connected together by a linear torsional spring $k$. Each pendulum is subjected to an external excitation $F \cos (\Omega \tau)$.

Using the Lagrangian approach, the equations of motion can be written as follows (Appendix A)

$$
m l^{2} \ddot{\phi}_{n}+\alpha l^{2} \dot{\phi}_{n}-k\left(\phi_{n-1}-2 \phi_{n}+k \phi_{n+1}\right)+m g l \sin \left(\phi_{n}\right)=F \cos (\Omega \tau) \quad n=1, \ldots, N,
$$

which represent a system of coupled nonlinear equations, subjected to an harmonic external excitation.

\subsection{Normalized equations}

For convenience and equation simplicity, the following nondimensional variables are introduced:

$$
t=\omega_{0} \tau \quad \theta_{n}=\frac{\phi_{n}}{\phi_{D}} \text { where } \phi_{D}=\frac{F \omega_{0}}{\alpha g l}, Q=\frac{\omega_{0} m}{\alpha} \text { and } \omega_{0}=\sqrt{\frac{g}{l}}
$$


Then, we obtain the following nondimensional system of equations

$$
\ddot{\theta}_{n}+\frac{1}{Q} \dot{\theta}_{n}+\frac{1}{\phi_{D}} \sin \left(\phi_{D} \theta_{n}\right)-\frac{k}{m g l}\left(\theta_{n-1}-2 \theta_{n}+\theta_{n+1}\right)=\frac{\alpha}{\omega_{0} m} \cos \left(\frac{\Omega}{\omega_{0}} t\right) .
$$

Equation (3) represents the normalized nonlinear differential system of a coupled pendulum array, where each pendulum is subjected to an external harmonic excitation. Although this equation can be solved numerically to provide standard reference, the branches resulting from the collective dynamics in the frequency domain will not be identified in terms of modal interactions and bifurcation topologies. To do so, in the following section, we are interested in an analytical solving procedure, enabling the identification of the solution branches type. Therefore, the full model will be replaced by a truncated one valid for small rotational motion..

The above EOMs system can be written in the following matrix form by expanding $\sin \left(\phi_{D} \theta_{n}\right)$ into its Taylor series:

$$
\underbrace{\mathbb{M} \ddot{\Theta}+\mathbb{C} \dot{\Theta}+\mathbb{K}_{L} \Theta}_{\text {Linear part }} \underbrace{+F_{N L}(\theta)}_{\text {Nonlinear part }}=F(t),
$$

with the rotating vector $\Theta=\left[\theta_{1}, \theta_{2}, \ldots, \theta_{N}\right]^{T}$, the excitation vector $\mathbb{F}(t)=\frac{\alpha}{\omega_{0} m} \cos \left(\frac{\Omega}{\omega_{0}} t\right)[1, \ldots, 1]^{T}, \mathbb{M}=\operatorname{diag}(1,1, \ldots, 1)$, $\mathbb{C}=\frac{1}{Q} * \operatorname{diag}(1,1, \ldots, 1), F_{N L}(\Theta)$ is the nonlinear stiffness vector and

$$
\begin{aligned}
& \mathbb{K}_{L}=\left(\begin{array}{ccccc}
1+\frac{2 k}{m g l} & -\frac{k}{m g l} & & & 0 \\
-\frac{k}{m g l} & 1+\frac{2 k}{m g l} & -\frac{k}{m g l} & & 0 \\
0 & \ddots & \ddots & \ddots & \\
& & -\frac{k}{m g l} & 1+\frac{2 k}{m g l} & -\frac{k}{m g l} \\
& & & -\frac{k}{m g l} & 1+\frac{2 k}{m g l}
\end{array}\right) \\
& N=1: \quad \omega_{1}=\sqrt{1+\frac{2 k}{m g l}} \\
& N=2: \quad \omega_{1}=\sqrt{1+\frac{k}{m g l}} \\
& \omega_{2}=\sqrt{1+\frac{3 k}{m g l}} \\
& N=3: \quad \omega_{1}=\sqrt{1-\frac{\sqrt{2} k}{m g l}+\frac{2 k}{m g l}} \\
& \omega_{2}=\sqrt{1+\frac{2 k}{m g l}} \\
& \omega_{3}=\sqrt{1+\frac{\sqrt{2} k}{m g l}+\frac{2 k}{m g l}}
\end{aligned}
$$

We may express all normal frequencies relative to the same reference frequency which is 1 , so that 


$$
\omega_{n}=\sqrt{\left(1+\lambda_{n} \Delta\right)} \quad(n=1, \ldots, N)
$$

where $\Delta=\frac{k}{m g l}$. We suppose that the pendulums are weakly coupled; so that $k<<m g l$. Consequently, $\Delta<<1$ and:

$$
\omega_{n} \approx 1+\frac{1}{2} \Delta \quad(n=1,2, \ldots, N)
$$

This assumption leads to the creation of linear closed modes which permit to study the effects of the mode localization on the collective dynamics. Several techniques have been developed to solve similar resulting nonlinear differential system (3), we can mention time integration, the shooting method [23], method of nonlinear normal forms [24], the homotopy analysis method [25] or the harmonic balance method (HBM) [26] coupled with the asymptotic numerical method (ANM) [27]. However, these methods are time consuming and not suitable for solving large size nonlinear systems. In particular, we focus on the multiple scales method [28] as an approximate analytical method to solve the nonlinear differential system, followed by numerical simulations to perform solutions of the resulting complex algebraic equations. The main advantage of the present approach is its capacity to handle weakly coupled nonlinear systems, which permits to visualize all physical responses branches and their properties in terms of modal interactions and bifurcation topology transfer.

\section{Solving procedure}

As we are interested in small rotational motion, we would expect to get a satisfactory approximation by expanding the nonlinear term $\sin \left(\phi_{D} \theta_{n}\right)$ into its Taylor series up to the third order by writing

$$
\sin \left(\phi_{D} \theta_{n}\right) \approx \phi_{D} \theta_{n}-\frac{1}{6} \phi_{D}^{3} \theta_{n}^{3}
$$

If we replace this approximation in the exact normalized differential equation (3), we get the following approximate equation

$$
\ddot{\theta}_{n}+\frac{1}{Q} \dot{\theta}_{n}+\theta_{n}-\frac{k}{m g l}\left(\theta_{n-1}-2 \theta_{n}+\theta_{n+1}\right)-\frac{1}{6} \frac{F^{2}}{\alpha^{2} g l^{3}} \theta_{n}^{3}=\frac{\alpha}{\omega_{0} m} \cos \left(\frac{\Omega}{\omega_{0}} t\right) .
$$

We shall solve Equations (8) using the multiple-scale perturbation theory, by introducing the appropriate parameters and setting the external frequency an amount $\varepsilon \omega_{0} \Omega_{D}$ away from the resonant frequency, so they can contribute to the amplitudes equations.

$$
\frac{1}{Q}=\varepsilon c, \quad \frac{k}{m g l}=\frac{1}{2} \varepsilon \gamma, \quad \frac{1}{6} \frac{F^{2}}{\alpha^{2} g l^{3}}=\xi \text { and } \frac{\alpha}{\omega_{0} m}=\varepsilon^{\frac{3}{2}} f .
$$

We express the solution of Equations (3) as a sum of standing-wave modes with slowly varying amplitudes, with fixed boundary conditions $\left(\theta_{0}=\theta_{N+1}=0\right)$ 


$$
\begin{aligned}
\theta_{n}(t)= & \varepsilon^{\frac{1}{2}} \sum_{m=1}^{N}\left(A_{m}(T) \sin \left(n q_{m}\right) e^{i t}+c . c .\right) \\
& +\varepsilon^{\frac{3}{2}} \theta_{n}^{(1)}(t)+\cdots, \quad n=1, \cdots, N,
\end{aligned}
$$

where $T=\varepsilon t$ is a slow time variable. The possible wave components $q_{m}$ can be given as

$$
q_{m}=\frac{m \pi}{N+1}
$$

We substitute the trial solution (10) into the normalized EOM term by term and we cancel order $\varepsilon^{\frac{1}{2}}$ and at order $\varepsilon^{\frac{3}{2}}$ we get $N$ equation of the form

$$
\ddot{\theta}_{n}^{(1)}+\theta_{n}^{(1)}=\sum_{m}\left(m^{t h} \text { secular term }\right) e^{i t}+\text { other terms }
$$

In the method of multiple scales, the amplitudes are allowed to vary slowly so to render the series expansions uniformly valid at large time. This can be done by eliminating the secular terms that cause unbounded perturbations. To extract the equation for the $m^{\text {th }}$ amplitude $A_{m}(T)$, we make use of the orthogonality of the modes, by multiplying the $m^{\text {th }}$ secular term by $\sin \left(n q_{m}\right)$ and summing over $n$. We find the coefficient of the $m^{\text {th }}$ secular term, which is required to vanish is given by

$$
2 i \frac{d A_{m}}{d T}+i c A_{m}+2 \gamma \sin ^{2}\left(\frac{q_{m}}{2}\right) A_{m}-\frac{3}{4} \xi \sum_{j, k, l} A_{j} A_{k} A_{l}^{*} \Delta_{j k l, m}^{(1)}=\frac{f}{N+1} e^{i \Omega_{D} T} \sum_{n=1}^{N} \sin \left(n q_{m}\right)
$$

Neglecting initial transients, we try a steady-state solution of the form

$$
A_{m}=a_{m} e^{i \Omega_{D} T} \text { with } a_{m}=\alpha_{m}+i \beta_{m}
$$

Substituting Equation (14) into Equation (13), we obtain the required equation for the complex amplitudes $a_{m}$.

$$
\left(i c-2 \Omega_{D}\right) a_{m}+2 \gamma \sin ^{2}\left(\frac{q_{m}}{2}\right) a_{m}-\frac{3}{4} \xi \sum_{j, k, l} a_{j} a_{k} a_{l}^{*} \Delta_{j k l, m}^{(1)}=\frac{f}{N+1} \sum_{n=1}^{N} \sin \left(n q_{m}\right)
$$

The differential system (13) of rotational dof $\phi_{n}(t)$ has been replaced using perturbation calculations by a time independent mode amplitudes $a_{m}$ system of coupled complex equations. All that remains, in order to study the collective dynamics of an array of coupled pendulums as a function of the original design parameters, is to solve these algebraic coupled complex equations. 


\section{Numerical and analytical studies}

Before starting our investigations, we should note that the second member of Equation (15) is proportional to the sum of standing waves, which is null for all even modes regardless of the considered number of coupled pendulums. Therefore, all even modes $a_{2 n}$ are not excited after modes projection, as they possess null trivial solutions. In addition, if we consider that whenever for a given mode $m, \Delta_{m m m ; j}^{(1)}=0$ for all $j \neq m$, then a Single Mode (SM) solution branch can exist with $a_{m} \neq 0$ and $a_{j}=0$. Thus, the only SM solution can exist for the first mode $a_{1}$ in the case of two coupled pendulums, where its amplitude takes the form of a single driven Duffing oscillator response.

For the rest of the paper we will consider the design parameters listed in the following Table 1, which satisfies the modes localization assumption given in Equation (6).

Table 1: Design parameters for the corresponding periodic structure depicted in Figure 1
\begin{tabular}{|c|c|c|c|c|c|}
\hline $\mathrm{m}(K g)$ & $\mathrm{g}\left(m . s^{-2}\right)$ & $1(m)$ & $\mathrm{k}(N . m)$ & $\alpha\left(K g . s^{-1}\right)$ & $F(N . m)$ \\
\hline 0.25 & 9.81 & 0.062 & 0.0009 & 0.16 & 0.01 \\
\hline
\end{tabular}

Note that, since the considered system is periodic and since we expressed the solution as a sum of standing wave modes with slowly varying amplitude (10), nearly symmetric responses were obtained. Thereby, for the rest of the paper we choose to plot the intensity responses of the rotational dofs $\left|\phi_{i}\right|$ for $\mathrm{n} \in 1, \ldots, E\left(\frac{N+1}{2}\right)$.

\subsection{Three coupled pendulums}

In order to investigate the modal interactions and their effects on the nonlinear dynamics of a periodic coupled pendulums chain, we consider the case of three coupled pendulums under periodic external harmonic excitation. Before doing so, one must verify qualitatively as well as quantitatively with a brute numerical simulation the validity and the reliability of the proposed analytical-numerical method.

Concerning numerical simulations, a direct time integration method using Runge-Kutta algorithme has been employed in order to solve the approximate differential system (1). However, the resulting algebraic nonlinear system (15) of the employed analytical procedure has been solved using a Newton-Raphson algorithm in Mathematica. For several values of the detuning parameter $\Omega_{D}$ inside the frequency range, Mathematica represents whole dynamic responses. The stability of the different solution branches has been performed based on the Eigen values of the Jacobian matrix of the differential system (13) computed numerically for each point. Figure 2 shows the rotational dofs of three coupled pendulums as a function of frequency for the design parameters listed in Table 1. The error between both methods rise to $5 \%$ on the lower branches. As perturbation methods are well adapted to small damped nonlinear systems; the error decrease by decreasing the damping coefficient. 

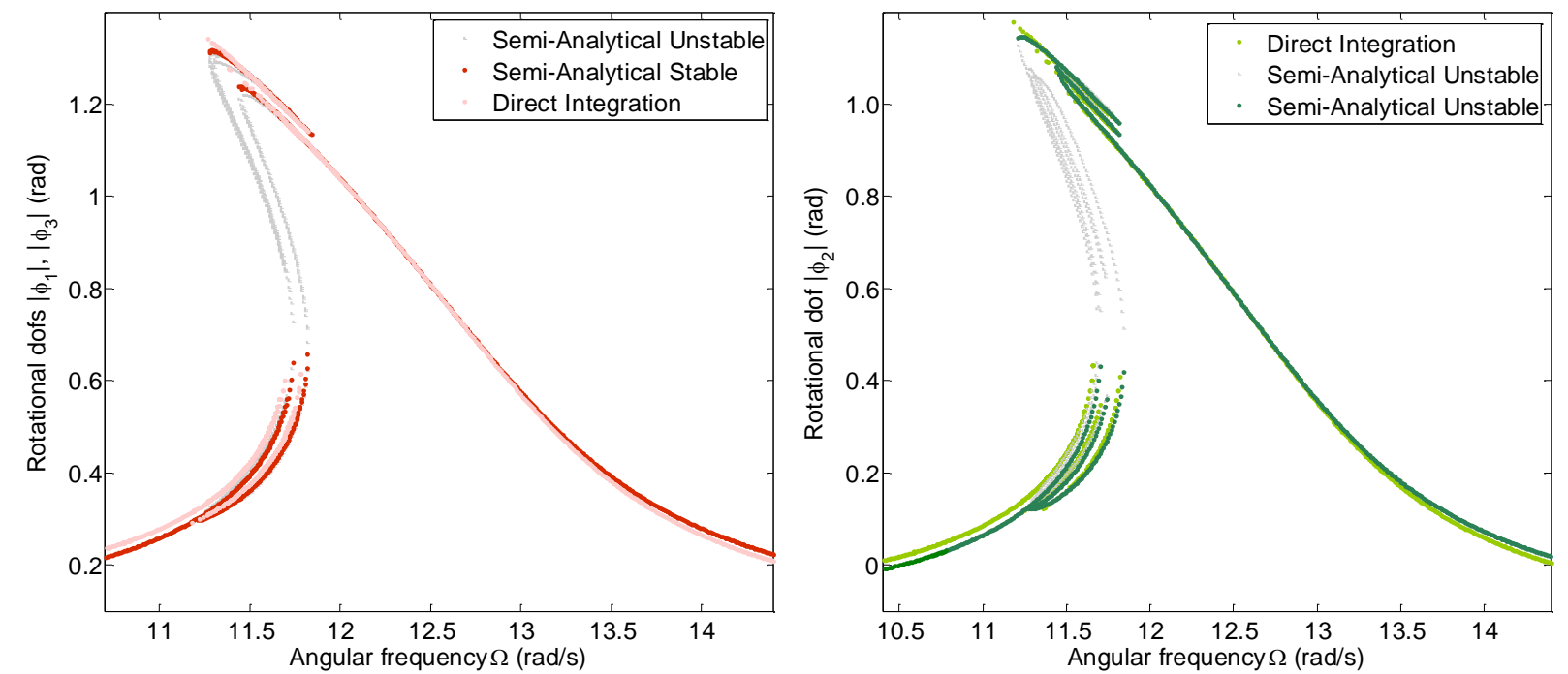

Figure 2: Rotational dofs of three coupled pendulums as a function of frequency for the design parameters listed in Table 1. Comparison between a direct time integration method using Runge-Kutta which is employed to solve the approximate differential system (A.7), and the semi-analytical method.

Direct time integration methods present an easy implementation procedure to plot nonlinear frequency response curves, considering a numerical step-by-step procedure. However, once the multistability domain is reached, jump phenomena may occurs according to the initial conditions that have been taken into account. Consequently, these methods may not give enough information about the global dynamics of the system, particularly the bifurcation behavior where they generally fail to capture unstable solutions and they are not able to identify the nature of multimode branches anyhow. In addition, they are time consuming, since they require highly computational time especially when considering strongly nonlinear high order systems subjected to weak damping.

The employed perturbation technique is based on expanding the periodic solution in the form of a power series in order to obtain an approximate analytical solution of the system. It allows a detailed study regarding the stability, the type of solution branches in term of modal interactions and bifurcation topology transfer. Thus, the proposed solving procedure is robust and efficient to investigate the collective dynamics of weakly coupled nonlinear periodic structures.

Symbolic computations were performed in order to solve the complex algebraic system (15) for three coupled pendulums $(N=3)$ under external excitation, based on prediction-correction algorithms. The natural dimensionless eigenfrequencies of the associated linear system are $\omega_{1}=1.0017, \omega_{2}=1.0059$ and $\omega_{3}=1.01$. In addition, the generalized modal forces vector $X^{T} F$ is $[0.017,0,-0.029]$, which means that the second mode is not excited and does not contribute to the dynamic responses.

In Figures 3 (a), we show the solutions for the response intensity of three coupled pendulums under external excitations, as a function of frequency. Branches labeled DMi represent the Double Mode solutions branches involving 
the excitation of odd modes $\left(a_{1} \neq 0, a_{3} \neq 0\right.$ and $\left.a_{2}=0\right)$, where $T M j$ represent the Triple Mode solutions generated by all modes collectively. For three coupled pendulums all solution branches are multimodal where we can obtain up to six stable solutions for a given frequency. Note that each bifurcation point due to a multimodal solution has a correspondence on the two other intensity responses. These curves were plotted to underline the large number of stable solution branches, even for a small number of coupled pendulums.

As the number of multimodal solution branches is important, we analyze each single branch separately to explore the practical appearance of each attractor. Therefore, we make use of the basins of attraction to demonstrate the robustness of these solutions. Figure 3 (b) show the basins of attraction in the Nyquist plane $\left(\alpha_{1}(0), \beta_{1}(0)\right)$ for $f=$ $1.83 \mathrm{~Hz}$ and a random configuration of initial conditions $\alpha_{2}(0)=0.1, \beta_{2}(0)=0, \alpha_{3}(0)=0.5$ and $\beta_{3}(0)=-0.3$. We can notice that we have basins of attraction topology transfer between odd modes $\left|a_{1}\right|^{2}$ and $\left|a_{3}\right|^{2}$ with respect to the solution branch nature, due to the modal interaction between them. In addition, the distribution of the DM solution branches corresponds to the distribution of the null trivial solution of $\left|a_{2}\right|^{2}$. Although, safe basins and integrity measures $[29,30]$ do not take part in this study, they must be thoroughly and deeply analyzed by choosing the right definition of safe basin, for a complete overview on this topic.

In addition, as the even modes are not excited a ROM approach considering odd modes only has been proposed in order to reduce the CPU time. Figure 4 shows the rotational dofs $\phi_{n}$ as a function of frequency comparing results obtained when considering the full model and when applying the ROM approach. For a single pendulum the response intensity corresponds to a forced frequency response of a single Duffing oscillator, however for the three weakly coupled pendulums, we observe additional solution branches resulting from the interaction between localized modes. Starting from the response intensities plotted in Figure 3, to the rotational dofs given in Figure 4, one can remark that the multimodal solutions were distributed in the multistability domain in a manner to join either resonant or non-resonant branches. 

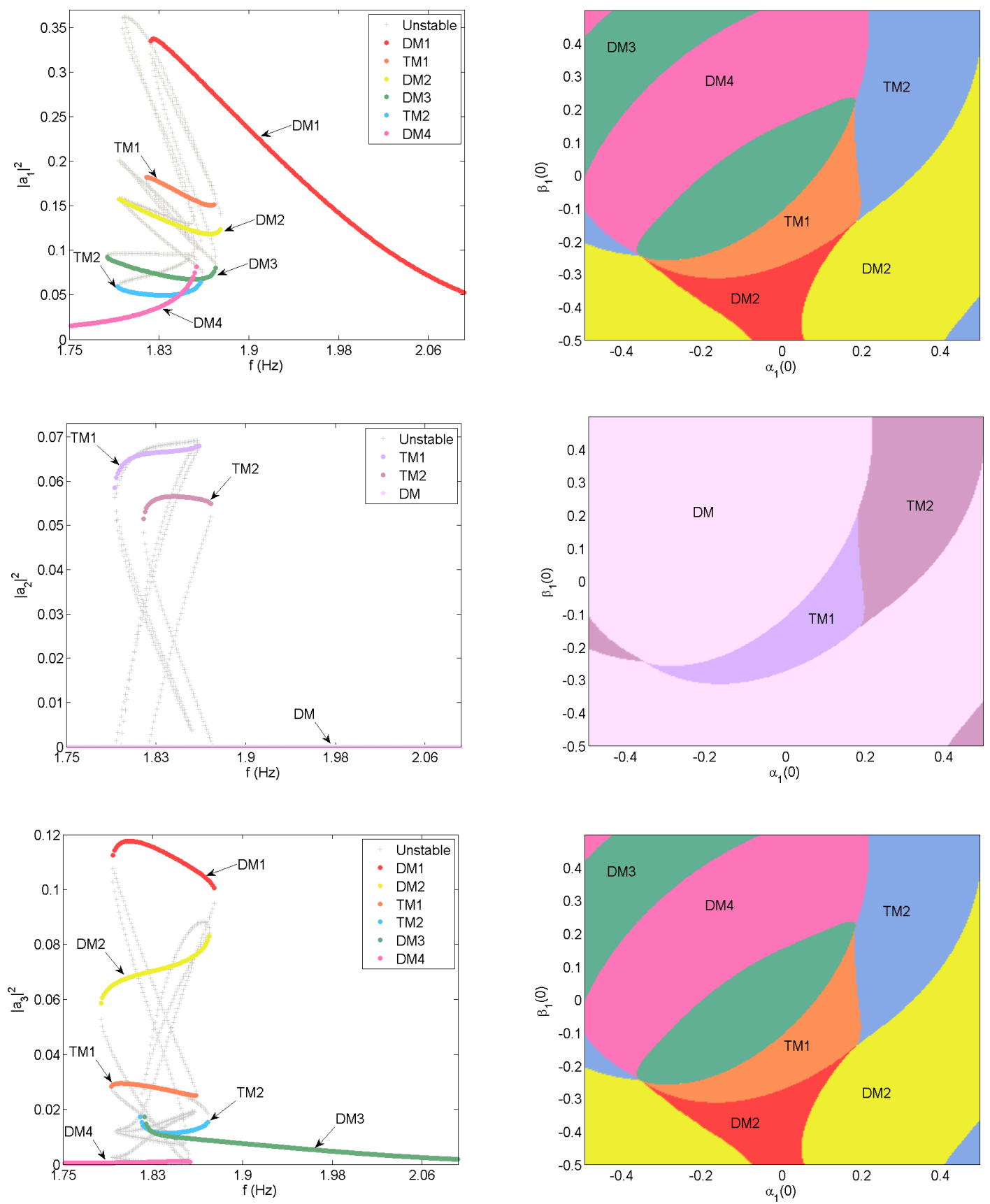

(a)

(b)

Figure 3: (a) Response intensity of three coupled pendulums under external excitation as a function of frequency. Solid curves indicate stable solutions and dashed curves indicate unstable solutions. DMi represent Double Modes solution branches, generated by the first and the thirst mode where the second one is null. $T M j$ are the Triple Mode solution branches involved by exciting all modes respectively. (b) Basins of attractions of these responses for $f=1.83 \mathrm{~Hz}$ in the Nyquist plane $\left(\alpha_{1}(0), \beta_{1}(0)\right)$ and random variable $\alpha_{2}=0.1, \beta_{2}=0, \alpha_{3}=0.5$ and $\beta_{3}=-0.3$. Each color reflects the distribution of a specific multimodal solution. 

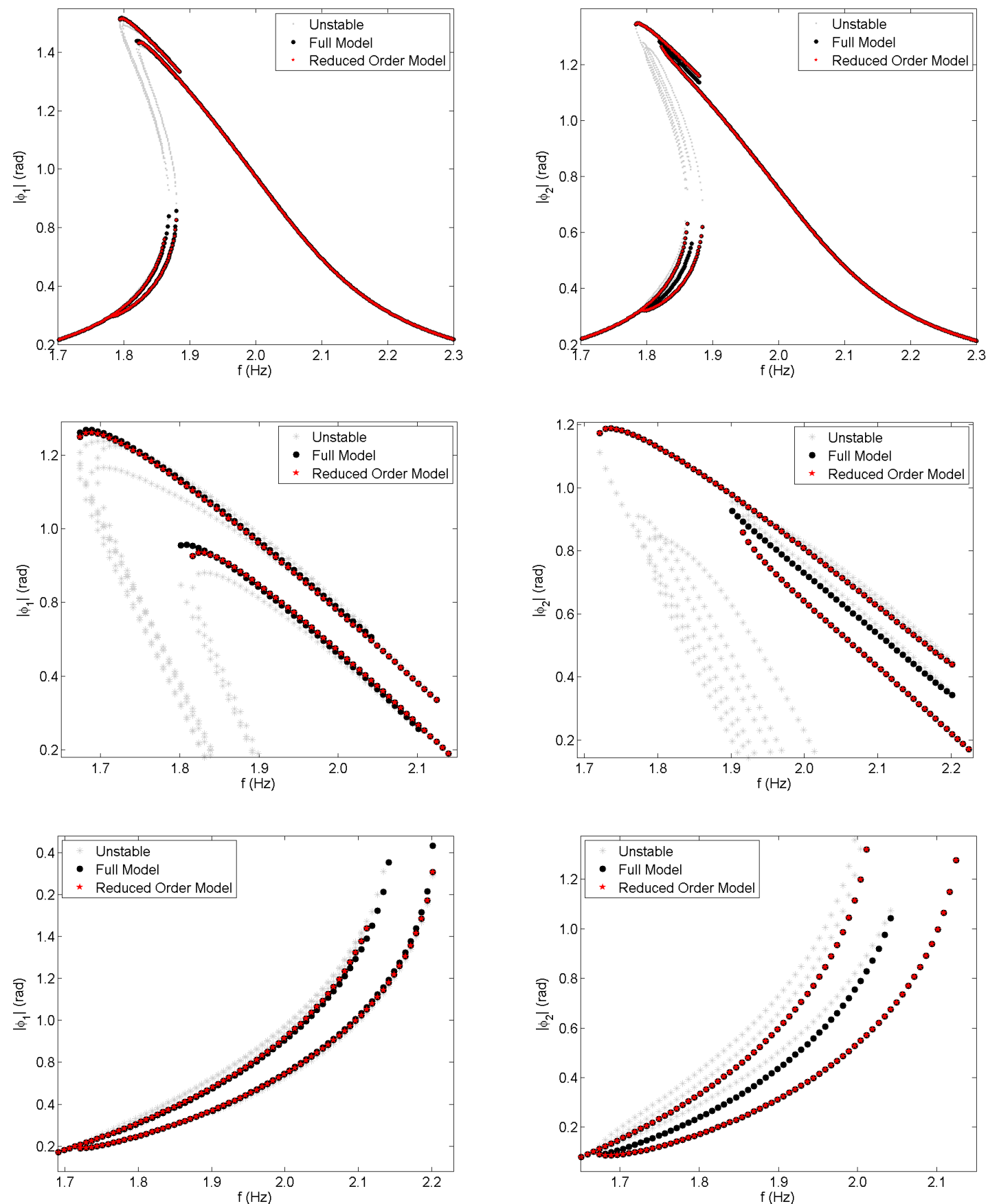

Figure 4: Rotational dof as a function of frequency of three coupled pendulums, where all pendulums are excited with external forces, where we zoom and highlight overs a couple of multimodal areas. Black, red and gray curves represent the full model, the reduced order model (The reduction basis contains odd modes only) and the stable branches. 

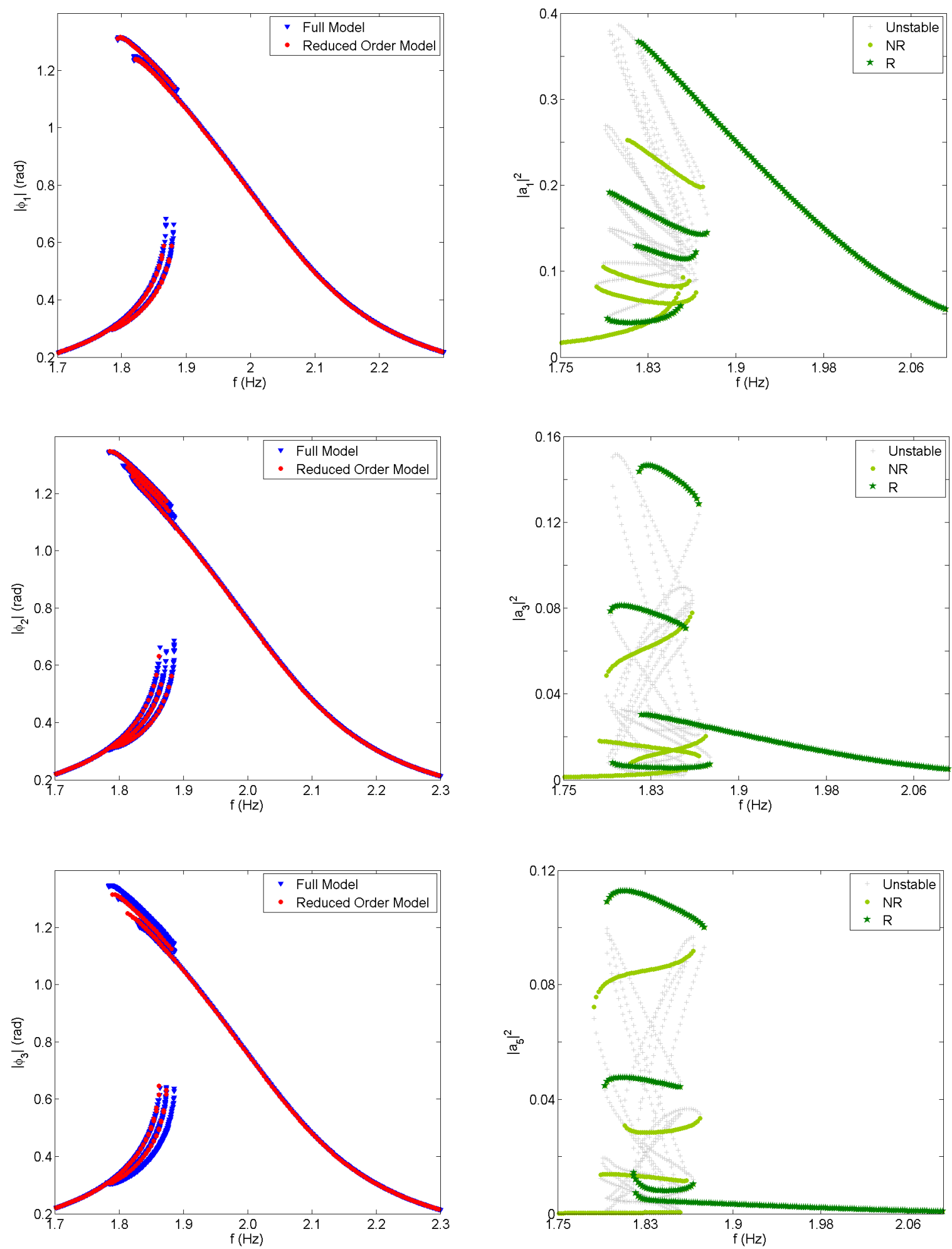

(a)

(b)

Figure 5: (a) Rotational dof as a function of frequency of six coupledlpendulums, where all pendulums are excited with external forces. Blue curves represent the full model and the Red curves represent the reduced order model (The reduction basis contains odd modes only). (b) Modal intensities of odd modes for the case of six coupled pendulums under external excitation after model order reduction as a function of frequency. Red, blue and gray curves represent the contribution of the TM solution branches in the frequency responses as Resonant (R), Non-Resonant (NR) and stable branches. 
Applying the proposed ROM approach goes to be cancelling the 2 nd mode $\left(a_{2}=0\right)$, therefore the rotations dofs consist only of DM solutions. The comparison between both approaches shows that the DM solutions capture almost all branches, while the TM (Full model) contributes with additional separated branches for the second rotational dof only. Consequently, as the absence of the $2^{\text {nd }}$ mode maintains the dominant dynamics without significant loss of accuracy compared to the full model and in order to reduce the CPU time, we propose to exclude it from the projection basis.

\subsection{Six coupled pendulums}

For larger number of coupled pendulums, symbolic computations become time-consuming, especially when visualizing the whole dynamics of the responses including the unstable branches. We may use an appropriate time integration procedure in order to solve the ordinary differential system (13), which is based on the fourth-order Runge Kutta integration method, allowing us to identify the nature of the branches with a low of computational time compared to the case when treating the initial differential system.

For the case of six coupled pendulums $(N=6)$, the natural dimensionless eigenfrequencies of the associated linear system are: $\omega_{1}=1.00117, \omega_{2}=1.00446, \omega_{3}=1.0092, \omega_{4}=1.01447, \omega_{5}=1.01922$ and $\omega_{6}=1.0225$. The generalized modal forces vector $X^{T} F$ is $[0.0234,0,0.0067,0,-0.0025,0]$, which means that the even modes are not excited after projecting on the standing wave modes. Then, the responses will take the form of a combination of two different types of modes: Triple Mode $(T M)$ resulting from the interaction between odd excited modes and sextuple mode $\left(6^{\text {th }} M\right)$ solution branches which is driven by the excitation of all modes collectively. As applying the ROM approach consists in writing the odd modes only in the reduction basis, all solution branches in the frequency responses each rotational dof will be of TM type.

Figure 5 (a) shows the rotational dofs as a function of frequency for six weakly coupled pendulums, where each pendulum is excited with an external force. Blue curves represent the full model $\left(T M+6^{\text {th }} M\right)$ and the Red curves represent the ROM (TM). Results of this model reduction show that the modal reduction preserves the accuracy of the full model in the prediction of all responses. Nevertheless, as the multimodal TM solutions join either resonant or non-resonant branches, further informations about the modal responses are required.

Therefore, we calculate the square of amplitudes of the odd modes in order to see the contribution of each triple mode solution branch in the rotational dof. Figure 5 (b) shows the response intensities of the odd modes as a function of frequency. Dark and light green curves represent the solutions that contribute as a Resonant (R) and Non-Resonant (NR) branches in the rotational dofs respectively. Remarkably, compared to the case of three coupled pendulums, the number of multi-modal solutions increases with up to eight possible solutions for a given frequency, for the case of six coupled pendulums even after applying the ROM approach. In addition, we can see that the multimodal solutions are distributed half-wave between $\mathrm{R}$ and NR in the rotational dofs. 

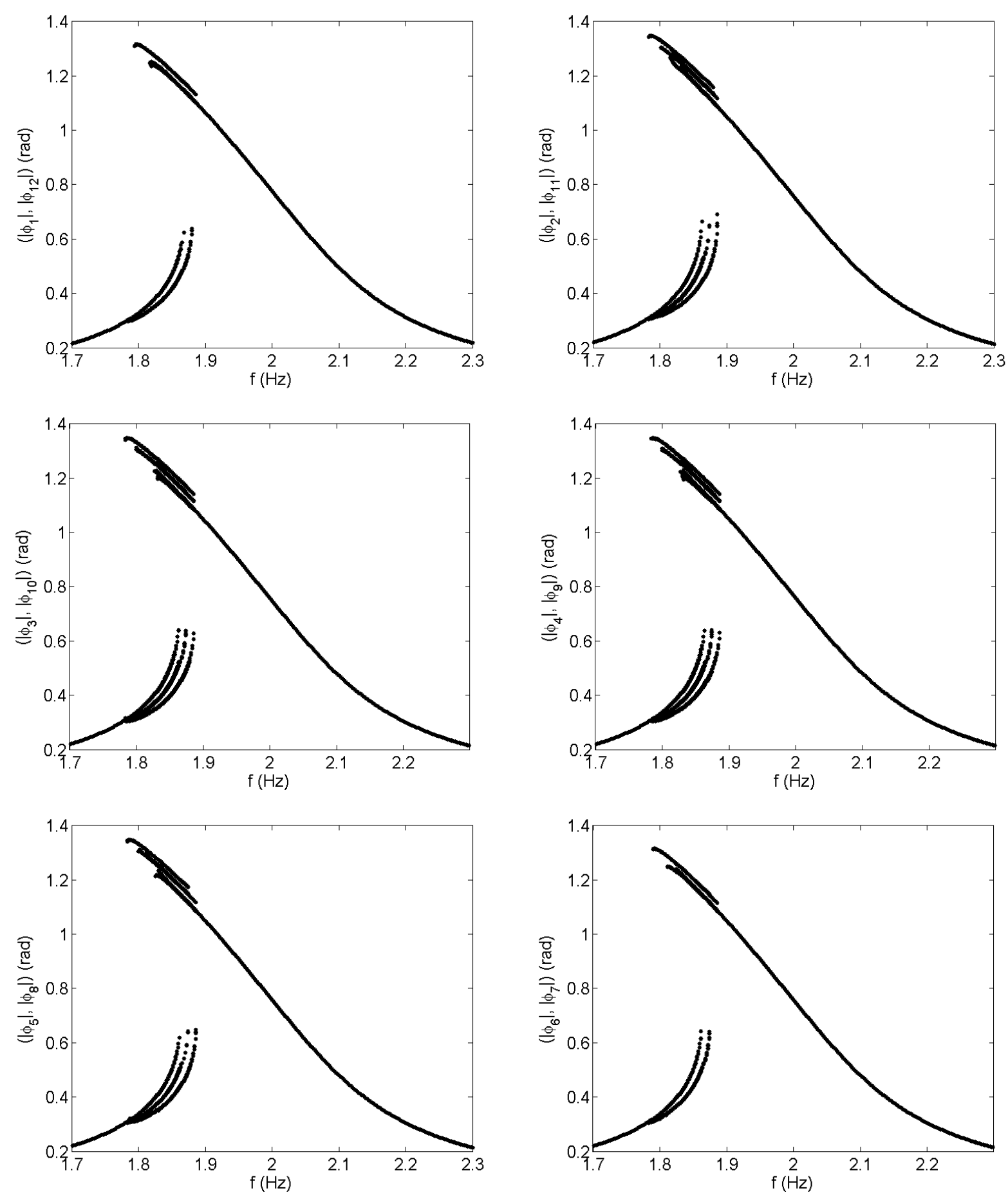

Figure 6: Rotational dof as a function of frequency of twelve coupled pendulums, where all pendulums are excited with external forces. Curves represent the reduced order model where the reduction basis contains odd modes only (6 modes). 
For $N$ weakly coupled pendulums, where each of these pendulums is subjected to an external harmonic excitation, the dimensionless eigenfrequencies of the associated linear system can be expressed as: $\omega_{m}=1+\varepsilon_{m}$ and the odd modes are not excited and do not contribute to the dynamic responses. The multi-mode solution branches of the corresponding response intensities are generated either by the excitation of odd modes or by all modes collectively. Then, a ROM approach based on using the even modes only can be applied, reducing the CPU time, while preserving the accuracy of the full model. In addition, the symmetry of the pendulums array leads to identical dynamics behavior of pairs of dofs $\left(\phi_{i}, \phi_{N+1-i}\right)$. As the number of coupled pendulums increases, the number of multi-modal solution branches increases and the system becomes more complex.

Figure 6 shows the rotational dofs of twelve coupled pendulums as a function of frequency, where the ROM approach was applied by only writing the odd modes in the reduction basis. The symmetry of the array of pendulums leads to similar dynamics behavior of six pairs of dof $\left(\phi_{1}, \phi_{12}\right),\left(\phi_{2}, \phi_{11}\right),\left(\phi_{3}, \phi_{10}\right),\left(\phi_{4}, \phi_{9}\right),\left(\phi_{5}, \phi_{8}\right)$ and $\left(\phi_{6}, \phi_{7}\right)$. The response frequencies of $N$ coupled pendulums have similar-trending curves, where the multimodal solutions join either R or NR branches in the rotational dofs. In order to highlight the benefits of treating large number of degreesof-freedom and extract some interesting features, a study of basins of attraction in the phase portrait can be performed for a given frequency in the multistability domain.

\subsection{Basins of attraction analysis}

Figure 7 displays the basins of attractions of $\left|\phi_{1}\right|$ for $f=1.83 \mathrm{~Hz}$ in the phase portrait $\left(\phi_{1}(0), \dot{\phi}_{1}(0)\right)$ for three, four, five and six coupled pendulums simultaneously. Red and blue colors denotes respectively the Resonant (R) and the Non-Resonant (NR) branches of the frequency response of the first dof $\left|\phi_{1}\right|$. As shown by the sequence of graphs, the distribution of the basins of attraction of the resonant branches (Red areas) increases slightly while increasing the number of coupled pendulums. Although, the study of basins of attraction in term of amplitude (R/NR) is important, we need to investigate the relationship between the type of these amplitudes and the nature of contributed modes.

Figure 8 shows the evolution of the basins of attractions in terms of type of branch and nature of mode of $\left|\phi_{1}\right|$ for $f=1.83 \mathrm{~Hz}$ in the phase portrait $\left(\phi_{1}(0), \dot{\phi}_{1}(0)\right)$ for three, four, five and six coupled pendulums simultaneously. Table 2 shows the color palette, where each color is related to a specific type of branche. $D M, T M, Q M, 5 M$ and $6 M$ are respectively the Double, Triple, Quadruple, Quintuple and Sextuple Modes.

From the previous diagrams, the basins of attractions of the DM are larger for $N=3$ than for $N=4$. Moreover the distribution of the TM in the case of three coupled pendulums $(N=3)$ covers small areas compared to the case of $N=5$; this distribution decreases for six coupled pendulums. Remarkably, the distribution of multimodal solution branches generated by all modes simultaneously increases while increasing the number of coupled pendulums. This can serve as a hint of the important distribution of resonant multimodal solutions, expected for large number of coupled pendulums. 


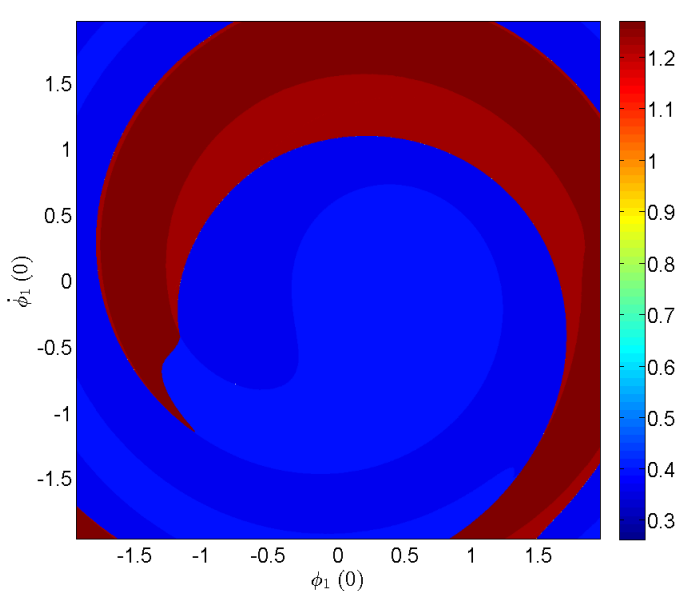

(a) Three coupled pendulums $(\mathrm{N}=3)$

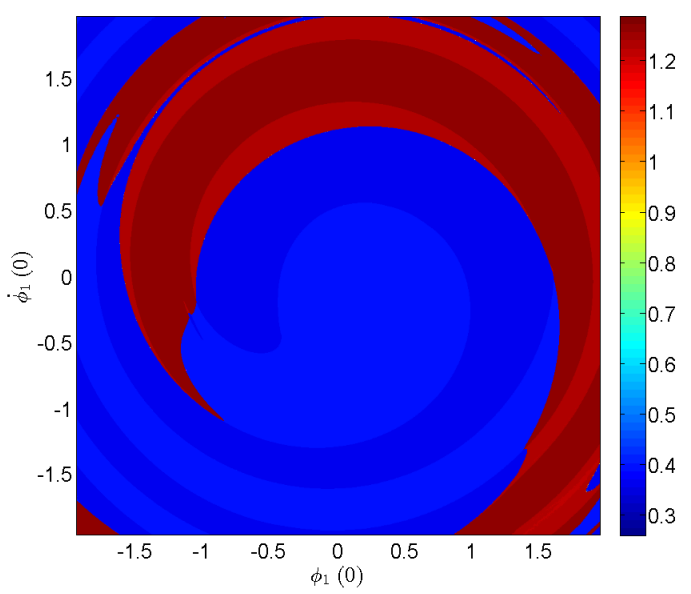

(c) Five coupled pendulums $(\mathrm{N}=5)$

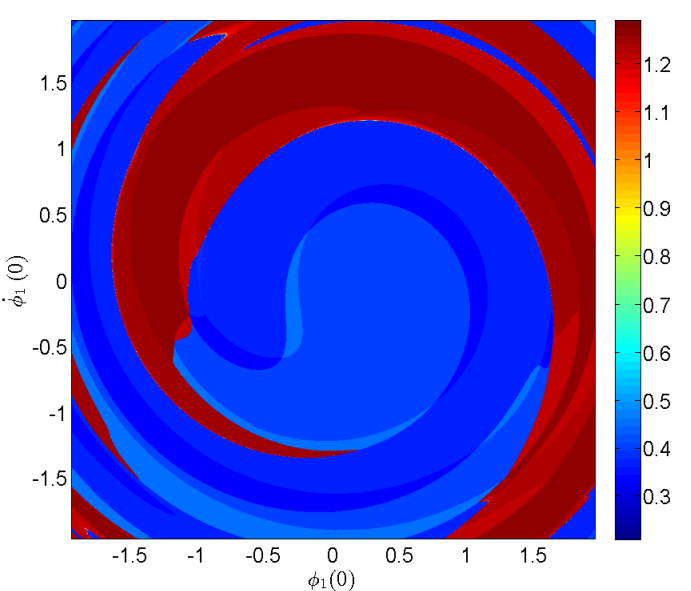

(b) Four coupled pendulums $(\mathrm{N}=4)$

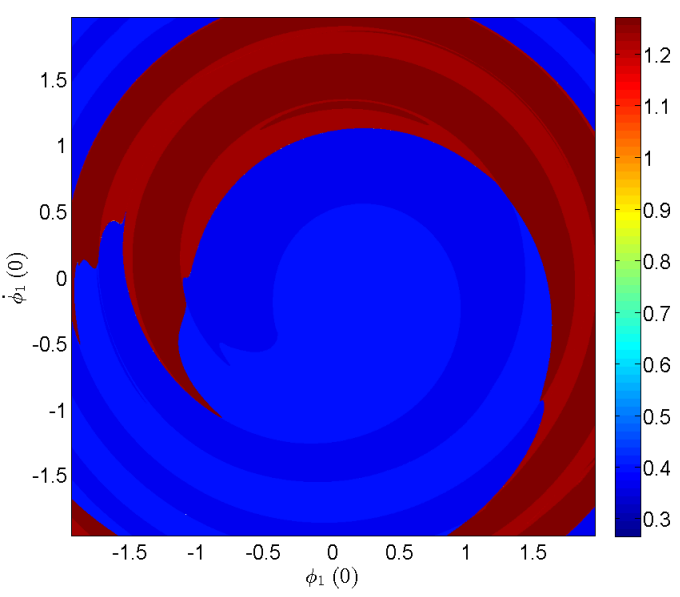

(d) Six coupled pendulums $(\mathrm{N}=6)$

Figure 7: Basins of attraction of $\left|\phi_{1}\right|$ for $f=1.83 \mathrm{~Hz}$ in the phase portrait $\left(\phi_{1}(0), \dot{\phi}_{1}(0)\right)$ for three, four, five and six coupled pendulums. Red and blue colors indicate respectively the Resonant $(\mathbf{R})$ and the Non-Resonant $(\mathbf{N R})$ branches in the frequency response of the first dof $\left|\phi_{1}\right|$. 


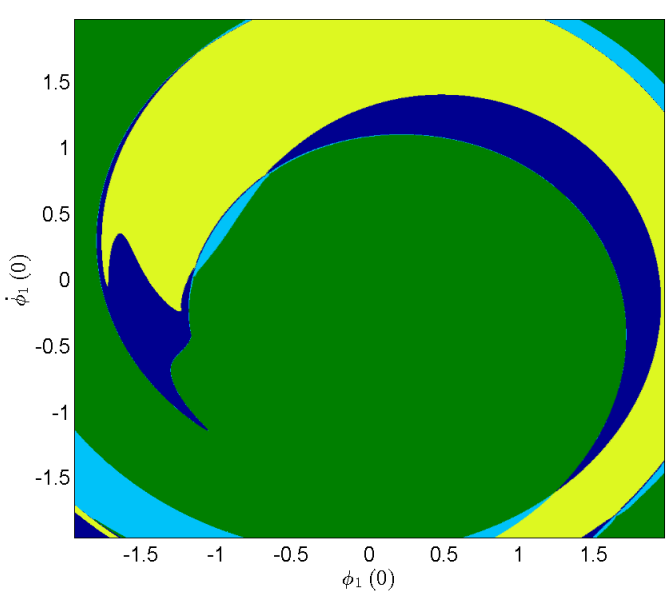

(a) Three coupled pendulums $(\mathrm{N}=3)$

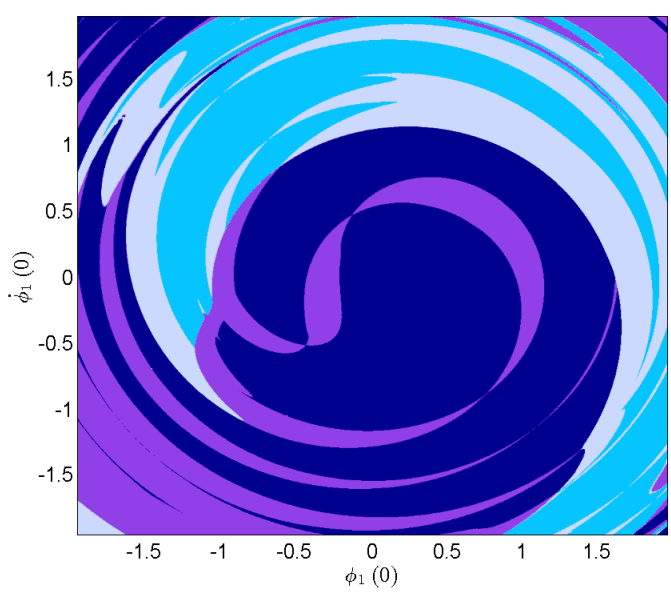

(c) Five coupled pendulums $(\mathrm{N}=5)$

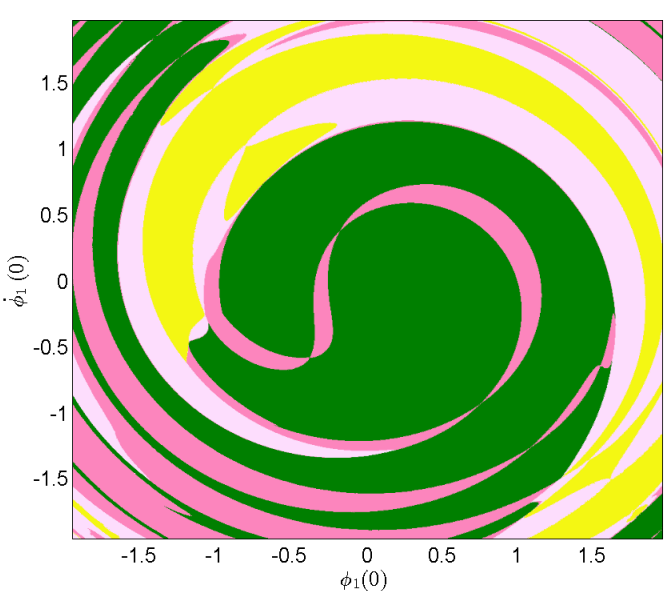

(b) Four coupled pendulums $(\mathrm{N}=4)$

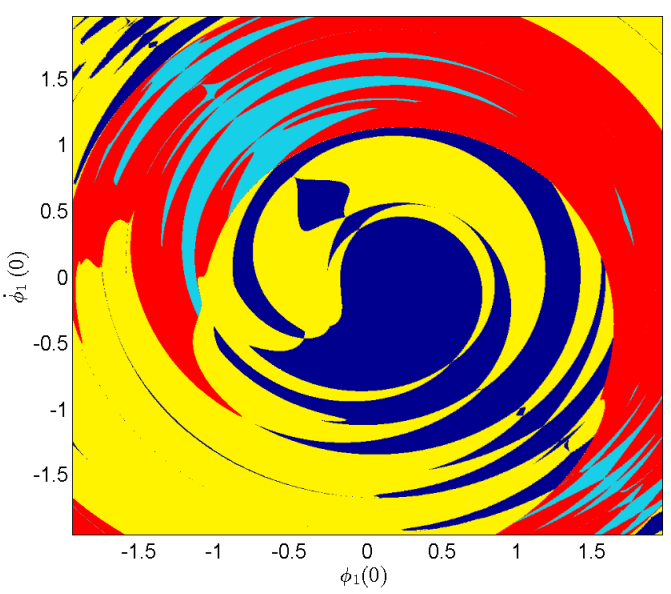

(d) Six coupled pendulums $(\mathrm{N}=6)$

Figure 8: Basins of attraction of $\left|\phi_{1}\right|$ for $f=1.83 \mathrm{~Hz}$ in the phase portrait $\left(\phi_{1}(0), \dot{\phi}_{1}(0)\right)$ for three, four, five and six coupled pendulums. Each color corresponds to different types of mode and branch, they are illustrated in Table 2.

Table 2: Color palette to distinguish the solutions in terms of type of mode and branch.

\begin{tabular}{||l|l|l|l|l|l|l|l|l|l|l||}
\hline Color & & & & & & & & & & \\
\hline \hline Mode & DM & DM & TM & TM & QM & QM & $5 M$ & $5 M$ & $6 M$ & $6 M$ \\
\hline Branch & R & NR & R & NR & R & NR & R & NR & R & NR \\
\hline
\end{tabular}




\section{Conclusions}

The collective nonlinear dynamics of a periodic array of coupled pendulums was modeled under harmonic external excitation. A semi-analytical method suitable for nonlinear localized modes problems has been considered, based on a perturbation technique, combined with a standing wave decomposition, transforming the normalized differential system into a set of coupled complex algebraic one. The resulting system has been numerically solved and the rotational dofs were plotted as a function of frequency to highlight the complexity and the multivaludness of the responses. In addition, the validity of the proposed semi-analytical method has been verified, and its role in identifying the type of the solution branches has been highlighted.

The choice of the physical parameters ensuring sufficiently weak substructure coupling, leads to strongly modes localization for perfectly periodic pendulums array. High number of multimodal solutions has been obtained for few coupled pendulums, resulting from the excitation of several modes collectively. The robustness of these additional branches has been proved with a classical study of their basins of attraction, as well as the bifurcation topology transfer. Similar dynamical behavior between frequency responses, resulting from the symmetry of the array has been identified. In addition, we notice that the multimodal solutions are distributed in a manner to join either resonant or non-resonant branches.

A model order reduction approach has been applied to solve large periodic arrays of coupled oscillators under external forces which helps reducing the CPU time. It is based on projecting on odd modes, while maintaining the dominant dynamics of the responses without significant loss of accuracy compared to the full model. The distributions of the basins of attraction of the resonant branches and the multimodal solutions governed by all modes simultaneously increase while increasing the number of coupled pendulums. In practice, this model could be used as a design tool to tune the number of multimodal solutions and increase the possibility to reach resonant branches. Consequently, while choosing the appropriate transaction technique [31], this model may lead to promising performances for energy scavenging applications.

Acknowledgments. This project has been performed in cooperation with the Labex ACTION program (contract ANR11-LABX-01-01).

\section{Appendix A. Equation of motion of $n^{\text {th }}$ pendulum}

The kinetic energy $T$ of the system can be expressed as

$$
T=\frac{1}{2} \sum_{n=1}^{N} m\left(\dot{x}_{n}^{2}+\dot{y}_{n}^{2}\right)
$$

Where $\left(x_{n}, y_{n}\right)$ represents the Cartesian coordinates system of the $n^{\text {th }}$ pendulum and the dot denotes the derivative with respect to time, $x_{n}$ and $y_{n}$ are given as 


$$
x_{n}=l \sin \phi_{n}, \quad y_{n}=l \cos \phi_{n} .
$$

by substituting Equation (A.2) into Equation (A.1) the kinetic energy becomes

$$
T=\frac{1}{2} \sum_{n=1}^{N} m l^{2} \dot{\phi}_{n}^{2}
$$

The potential energy $V$ of the system can be written as

$$
V=\sum_{n=1}^{N} m g l\left(1-\cos \phi_{n}\right)+\frac{1}{2} \sum_{n=1}^{N-1} k\left(\phi_{n+1}-\phi_{n}\right)^{2} .
$$

Where $g$ represents the acceleration due to gravity. The dissipation energy $D$ of the system is given by

$$
D=\frac{1}{2} \sum_{n=1}^{N} \alpha l^{2} \dot{\phi}_{n}^{2}
$$

where $\alpha$ is the viscous damping coefficient. The Lagrange equations can be written in the following form

$$
\frac{d}{d \tau}\left(\frac{\partial L}{\partial \dot{\phi}_{n}}\right)-\frac{\partial L}{\partial \phi_{n}}+\frac{\partial D}{\partial \dot{\phi}_{n}}=Q_{n} \quad n=1, \ldots, N,
$$

With $L$ the Lagrangian operator defined by $L=T-V$ and $Q_{n}$ relative to external forces. Substituting equations (A.3), (A.4) and (A.5) into the Lagrange's equation (A.6) leads to the following differential equation of the $n^{\text {th }}$ pendulum

$$
m l^{2} \ddot{\phi}_{n}+\alpha l^{2} \dot{\phi}_{n}+m g l \sin \left(\phi_{n}\right)-k\left(\phi_{n-1}-2 \phi_{n}+\phi_{n+1}\right)=F \cos (\Omega \tau) \quad n=1, \ldots, N,
$$

This expression is expressed in the manuscript by Equation (1). Expanding $\sin \left(\phi_{n}\right)$ as the sum of its Taylor series up to the third order, leads to the EOM of the $n^{\text {th }}$ pendulum (8) for small rotations.

Figure A.9 (a) shows the comparison of the response frequency of one pendulum $(\mathrm{N}=1)$ using direct time integration method between the full nonlinear problem (A.7) and the one obtained after applying Taylor series (8), for the design parameters listen in Table 1. Equation (8) gives a good approximation of the full nonlinear integration for which the error does not exceed 3\%. This result is confirmed in Figure A.9 (b) where we compare the function $\sin (\phi)$ and its Taylor series as a function of the rotational amplitude $\phi$ up to $1.4 \mathrm{rad}$. 


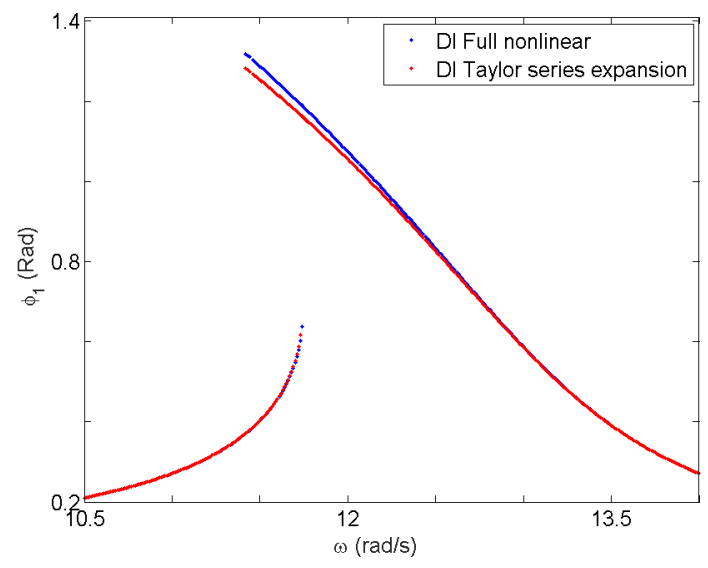

(a)

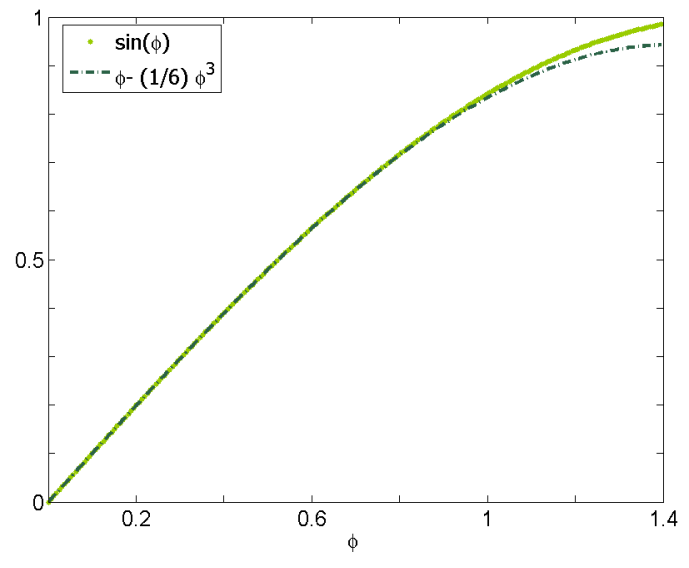

(b)

Figure A.9: (a) Response frequency for one pendulum $(\mathrm{N}=1)$ using direct time integration method between the full nonlinear problem (A.7) and the one obtained after applying Taylor series (8). (b) Comparison between $\sin (\phi)$ and its Taylor series $\phi-\frac{1}{6} \phi^{3}$ as a function of the rotational amplitude $\phi$ up to 1.4 Rad.

\section{References}

[1] R. K. Narisetti, M. Ruzzene, M. J. Leamy, Study of wave propagation in strongly nonlinear periodic lattices using a harmonic balance approach, Wave Motion 49 (2) (2012) $394-410$.

[2] G. Heinrich, M. Ludwig, J. Qian, B. Kubala, F. Marquardt, Collective dynamics in optomechanical arrays, Phys. Rev. Lett. 107 (2011) 043603.

[3] R. Lifshitz, M. C. Cross, Nonlinear Dynamics of Nanomechanical and Micromechanical Resonators, Wiley-VCH Verlag GmbH and Co. KGaA, 2009, pp. 1-52.

[4] J. Cuevas-Maraver, P. Kevrekidis, F. Williams, The sine-Gordon Model and its Applications: From Pendula and Josephson Junctions to Gravity and High-Energy Physics, Nonlinear Systems and Complexity, Springer, 2014.

[5] J. A. Marlin, Periodic motions of coupled simple pendulums with periodic disturbances, International Journal of Non-Linear Mechanics 3 (4) (1968) $439-447$.

[6] D. Cai, A. R. Bishop, A. Sánchez, Length-scale competition in the damped sine-gordon chain with spatiotemporal periodic driving, Phys. Rev. E 48 (1993) 1447-1452.

[7] A. L. Fradkov, B. Andrievsky, Synchronization and phase relations in the motion of two-pendulum system, International Journal of NonLinear Mechanics 42 (6) (2007) 895 - 901.

[8] A. Najdecka, T. Kapitaniak, M. Wiercigroch, Synchronous rotational motion of parametric pendulums, International Journal of Non-Linear Mechanics 70 (2015) $84-94$

[9] T. Witelski, L. Virgin, C. George, A driven system of impacting pendulums: Experiments and simulations, Journal of Sound and Vibration 333 (6) (2014) $1734-1753$

[10] H. N. Huynh, T. P. T. Nguyen, L. Y. Chew, Numerical simulation and geometrical analysis on the onset of chaos in a system of two coupled pendulums, Communications in Nonlinear Science and Numerical Simulation 18 (2) (2013) 291 - 307.

[11] R. Khomeriki, J. Leon, Tristability in the pendula chain, Phys. Rev. E 78 (2008) 057202. 
[12] A. Vyas, A. Bajaj, Dynamics of autoparametric vibration absorbers using multiple pendulums, Journal of Sound and Vibration 246 (1) (2001) $115-135$.

[13] C. Liang, W. Ke, M. Fu, C. Wang, X. Chen, An undergraduate experiment of wave motion using a coupled-pendulum chain, American Journal of Physics 83 (5) (2015) 389-394.

[14] A. Jallouli, N. Kacem, N. Bouhaddi, Nonlinear dynamics of a 2d array of coupled pendulums under parametric excitation, in: 5th ECCOMAS Thematic Conference on Computational Methods in Structural Dynamics and Earthquake Engineering (COMPDYN 2015), 2015, p. 8 p.

[15] T. Ikeda, Y. Harata, K. Nishimura, Intrinsic localized modes of harmonic oscillations in pendulum arrays subjected to horizontal excitation, Journal of Computational and Nonlinear Dynamics 10 (2015) 021007.

[16] J. Cuevas, L. Q. English, P. G. Kevrekidis, M. Anderson, Discrete breathers in a forced-damped array of coupled pendula: Modeling, computation, and experiment, Phys. Rev. Lett. 102 (2009) 224101

[17] L. Q. English, Experimental Results for the sine-Gordon Equation in Arrays of Coupled Torsion Pendula, Springer, 2014, pp. 111-129.

[18] F. Geniet, J. Leon, Energy transmission in the forbidden band gap of a nonlinear chain, Phys. Rev. Lett. 89 (2002) 134102.

[19] R. B. Thakur, L. Q. English, A. J. Sievers, Driven intrinsic localized modes in a coupled pendulum array, Journal of Physics D: Applied Physics 41 (1) (2008) 015503.

[20] X. Hai-Qing, T. Yi, Parametrically driven solitons in a chain of nonlinear coupled pendula with an impurity, Chinese Physics Letters 23 (6) (2006) 1544.

[21] A. Jallouli, N. Kacem, N. Bouhaddi, Stabilization of solitons in coupled nonlinear pendulums with simultaneous external and parametric excitations, Communications in Nonlinear Science and Numerical Simulation 42 (2017) 1 - 11.

[22] D. Bitar, N. Kacem, N. Bouhaddi, M. Collet, Collective dynamics of periodic nonlinear oscillators under simultaneous parametric and external excitations, Nonlinear Dynamics 82 (1-2) (2015) 749-766.

[23] F. Wang, Bifurcations of nonlinear normal modes via the configuration domain and the time domain shooting methods, Communications in Nonlinear Science and Numerical Simulation 20 (2) (2015) 614 - 628.

[24] L. Stolovitch, Progress in normal form theory, Nonlinearity 22 (7) (2009) R77.

[25] S. Liao, Notes on the homotopy analysis method: Some definitions and theorems, Communications in Nonlinear Science and Numerical Simulation 14 (4) (2009) 983 - 997

[26] M. S. Hong-Hua Dai, S. N. Atluri, A simple collocation scheme for obtaining the periodic solutions of the duffing equation, and its equivalence to the high dimensional harmonic balance method: subharmonic oscillations, Computer Modeling in Engineering and Sciences 84 (5) (2012) 459.

[27] S.-L. Mei, C.-J. Du, S.-W. Zhang, Asymptotic numerical method for multi-degree-of-freedom nonlinear dynamic systems, Chaos, Solitons and Fractals 35 (3) (2008) $536-542$.

[28] A. Nayfeh, Introduction to Perturbation Techniques, Wiley Classics Library, Wiley, 2011.

[29] M. Soliman, J. Thompson, Integrity measures quantifying the erosion of smooth and fractal basins of attraction, Journal of Sound and Vibration 135 (3) (1989) 453 - 475.

[30] S. Lenci, G. Rega, Load carrying capacity of systems within a global safety perspective. part i. robustness of stable equilibria under imperfections, International Journal of Non-Linear Mechanics 46 (9) (2011) 1232 - 1239.

[31] P. Malaji, S. Ali, Analysis of energy harvesting from multiple pendulums with and without mechanical coupling, The European Physical Journal Special Topics 224 (14) (2015) 2823-2838. 